\title{
Korn Inequalities in Orlicz Spaces
}

\author{
MARTIN FUCHS
}

\begin{abstract}
We use gradient estimates for solutions of elliptic equations to obtain Korn's inequality for fields with zero trace from Orlicz-Sobolev classes.
\end{abstract}

As outlined for example in the monographs of Málek, Nečas, Rokyta, Růžička [18], of Duvaut and Lions [7] and of Zeidler [26], the wellposedness of many variational problems arising in fluid mechanics or in the mechanics of solids heavily depends on the positive answer to the following question: given a bounded domain $\Omega \subset \mathbb{R}^{n}, n \geq 2$, with Lipschitz boundary $\partial \Omega$ and a field $u: \Omega \rightarrow \mathbb{R}^{n}$ with zero trace, is it possible to bound a suitable energy norm (being determined by the variational problem under consideration) of the Jacobian matrix $\nabla u=\left(\partial_{\alpha} u^{i}\right)_{1 \leq \alpha, i \leq n}$ in terms of the norm of the symmetric gradient $\varepsilon(u):=\frac{1}{2}\left(\partial_{\alpha} u^{i}+\partial_{i} u^{\alpha}\right)_{1 \leq \alpha, i \leq n}$ ? Estimates of this form are known as Korn type inequalities, and the most elementary variant reads as follows: for any function $u$ from the Sobolev space $\stackrel{\circ}{W}_{2}^{1}\left(\Omega ; \mathbb{R}^{n}\right)$ (see Adams [2] for a definition) it holds

$$
\int_{\Omega}|\nabla u|^{2} d x \leq 2 \int_{\Omega}|\varepsilon(u)|^{2} d x .
$$

In fact, if $u$ denotes a smooth function with compact support in $\Omega$, then (1) can be obtained by partial integration, and the validity of (1) for Sobolev functions with zero trace is immediate. We note that $L^{2}$-variants of Korn's inequality go back to the works of Courant and Hilbert [5], Friedrichs [9], Èidus [8] and Mihlin [19].

Next we pass to the $L^{p}$-case with exponent $1<p<\infty$. Then it was shown by Gobert [Go1,2], Nečas [21], Mosolov and Mjasnikov [20], Temam [24] and later by the author [10] that there exists a

2000 Mathematics Subject Classification. 74B, 74G, 76D, 49J.

Key words and phrases. Korn inequalities, Orlicz-Sobolev spaces. 
positive constant $K=K_{p, n}(\Omega)$ depending on $p$ and the dimension $n$ as well as on the domain $\Omega$ such that the inequality

$$
\int_{\Omega}|\nabla u|^{p} d x \leq K \int_{\Omega}|\varepsilon(u)|^{p} d x
$$

is fulfilled for all $u \in \stackrel{\circ}{W} \underset{p}{1}\left(\Omega ; \mathbb{R}^{n}\right)$. A nice proof of $(2)$ is presented in Theorem 1.10, p.196, of [18] based on results of Nečas about equivalent norms on $L^{p}\left(\Omega ; \mathbb{R}^{n}\right)$ in terms of negative norms, which correspond to norms on the dual Sobolev space $\stackrel{\circ}{W}_{p}^{1}\left(\Omega ; \mathbb{R}^{n}\right)^{*}$.

Our short note now has been inspired by Remark 5 in the paper of Mosolov and Mjasnikov [20], where it is stated that "using the theorems of Simonenko [23] and Koizumi [15, 16] regarding the continuity of singular operators in Orlicz spaces, one can prove inequalities of the type of Korn's inequality in the corresponding spaces". To be precise, let us introduce the class $\Phi$ of all functions $\varphi:[0, \infty) \rightarrow[0, \infty)$, which are increasing and satisfy $\lim _{t \downarrow 0} \varphi(t)=0$, $\lim _{t \rightarrow \infty} \varphi(t)=\infty$.

Definition 1. A function $\varphi \in \Phi$ is a Young function if $\varphi$ is convex together with $\lim _{t \downarrow 0} \varphi(t) / t=\lim _{t \rightarrow \infty} t / \varphi(t)=0$.

Definition 2. Let $\varphi$ denote a Young function.

a) $\varphi$ is of type $(\Delta 2)$ if there is a constant $K>0$ such that $\varphi(2 t) \leq K \varphi(t)$ holds for all $t \geq 0$.

b) We define $\varphi$ to be of type $(\nabla 2)$ if for some constant $\widetilde{K}>1$ we have $\varphi(t) \leq \frac{1}{2 \widetilde{K}} \varphi(\widetilde{K} t), t \geq 0$.

Remark 1 . The $(\Delta 2)$ property - also known as doubling property of the Young function $\varphi$-guarantees that the Orlicz class $K_{\varphi}(\Omega)$ and the Orlicz space $L_{\varphi}(\Omega)$ coincide (see [2], Chapter VIII, for notation). Moreover, we can introduce the Orlicz-Sobolev space $\stackrel{\circ}{W}_{\varphi}^{1}\left(\Omega ; \mathbb{R}^{n}\right)$ of functions with zero trace in the usual way.

Remark 2. It should be noted that both $(\Delta 2)$ and $(\nabla 2)$ conditions make the Young function grow moderately. For example, if $\varphi$ is of class $C^{1}$, then $(\nabla 2)$ follows from the requirement that

$$
a(\varphi):=\inf _{t>0} \frac{t \varphi^{\prime}(t)}{\varphi(t)}>1
$$


is fulfilled. We refer the reader to the monograph of Roa and Ren [22], Corollary 4 on p.26.

With this notation we can give the following interpretation of Remark 5 from the paper [20].

Theorem 1. Let $\Omega$ be a bounded Lipschitz domain with small Lipschitz constants. Let $\varphi$ denote a Young function of type $(\Delta 2) \cap(\nabla 2)$. Then there is a constant $C=C_{\varphi, n}(\Omega)$ depending on $\varphi$, the dimension $n$ and the domain $\Omega$ such that

$$
\int_{\Omega} \varphi(|\nabla u|) d x \leq C \int_{\Omega} \varphi(|\varepsilon(u)|) d x
$$

is true for all fields $u \in \stackrel{\circ}{W}_{\varphi}^{1}\left(\Omega ; \mathbb{R}^{n}\right)$. In the case of two independent variables (3) can be replaced by

$$
\int_{\Omega} \varphi(|\nabla u|) d x \leq C \int_{\Omega} \varphi\left(\left|\varepsilon^{D}(u)\right|\right) d x,
$$

where $\varepsilon^{D}(u):=\varepsilon(u)-\frac{1}{n}(\operatorname{div} u) \mathbf{1}$ is the deviatoric part of $\varepsilon(u), \mathbf{1}$ denoting the unit matrix.

Remark 3. In their deep paper on stationary electrorheological fluids Acerbi and Mingione [1] prove variants of (3) for some special Young functions $\varphi$ (see Theorem 3.1 in this reference). Their argument is based on a kind of representation formula due to Ambrosio, Coscia and Dal Maso [3] (using in turn information from Kohn's thesis [14]) combined with an interpolation argument originating from Torchinsky's work [25].

Proof of Theorem 1. Our proof of (3) and (4) is based on gradient estimates in Orlicz spaces for solutions of elliptic equations recently obtained by Jia, Li and Wang [13]. Let $u$ denote a function from the class $C_{0}^{\infty}\left(\Omega ; \mathbb{R}^{n}\right)$, the general case follows by approximation. Then, as observed by Dain [6], we have the formula

$$
\Delta u^{j}=2 \partial_{i} \varepsilon^{D}(u)_{i j}-2\left(\frac{1}{2}-\frac{1}{n}\right) \partial_{j}(\operatorname{div} u), \quad j=1, \ldots, n,
$$

where the sum is taken with respect to indices repeated twice. We fix a coordinate direction $j \in\{1, \ldots, n\}$ and define $v:=u^{j}$,

$$
V:=\left(2 \varepsilon^{D}(u)_{i j}-2\left(\frac{1}{2}-\frac{1}{n}\right) \operatorname{div} u \delta_{i j}\right)_{1 \leq i \leq n} .
$$


Then, according to (5), $\Delta v=\operatorname{div} V$ and the desired inequalities (3) and (4) are a direct consequence of estimate (3.1) in Theorem 3.1 of [13], since on account of this reference we have the bound $\int_{\Omega} \varphi(|\nabla v|) d x \leq C \int_{\Omega} \varphi(|V|) d x$. Note that the domain $\Omega$ satisfies the assumptions from [13], as clarified in Remark 5 below.

Remark 4. We remark that Krylov [17] obtained Korn's inequality in the $L^{p}$-setting $(1<p<\infty)$ for fields equal to zero on the boundary by similar arguments as a result of his studies of the regularity properties of solutions to the Dirichlet problem for the Poisson equation in norms of negative order.

Remark 5. Of course the estimates (3) and (4) extend to the class of Reifenberg domains $\Omega \subset \mathbb{R}^{n}$ studied in the paper [13]. This follows from the comments given by Byun, Yao and Zhou stated after inequality (1.4) in their work [4], where it is said that domains with sufficiently small Lipschitz constants are $(\delta, R)$-Reifenberg flat.

Remark 6. As outlined in [13] and also discussed by Byun, Yao and Zhou [4] the basic gradient estimate for the Poisson equation with Orlicz space data holds if and only if the Young function $\varphi$ is of type $(\Delta 2) \cap(\nabla 2)$. This gives rise to the interesting question if $\varphi \in(\Delta 2) \cap(\nabla 2)$ is also a necessary condition for the validity of $(3)$ and (4).

\section{REFERENCES}

[1] E. Acerbi, G. Mingione, Regularity results for stationary electrorheological fluids, Arch. Rat. Mech. Anal. 164 (2002), 213-259.

[2] R. A. Adams, Sobolev spaces, Academic Press, New York-San FranciscoLondon (1975).

[3] L. Ambrosio, A. Coscia, G. Dal Maso, Fine properties of functions with bounded deformation, Arch. Rat. Mech. Anal. 139 (1997), 201-238.

[4] S. S. Byun, F. Yao, S. Zhou, Gradient estimates in Orlicz spaces for nonlinear elliptic equations, J. Funct. Anal. 255 (2008), 1851-1873.

[5] R. Courant, D. Hilbert, Methoden der mathematischen Physik, Vol. II, Springer Verlag, Berlin (1937).

[6] S. Dain, Generalized Korn's inequality and conformal Killing vectors, Calc. Var. 25 (4) (2006), 535-540.

[7] G. Duvaut, J. L. Lions, Inequalities in Mechanics and Physics, Springer Grundlehren 219, Springer Verlag, Berlin (1976).

[8] D. M. Èidus, On a mixed problem of the theory of elasticity, Dokl. Akad. Nauk SSSR 76 (1951), 181-184.

[9] K. Friedrichs, On the boundary value problems of the theory of elasticity and Korn's inequality, Annals of Math. (2) 48 (1947), 441-471. 
[10] M. Fuchs, On stationary incompressible Norton fluids and some extensions of Korn's inequality, Zeitschr. Anal. Anwendungen 13(2) (1994), 191-197.

[11] J. Gobert, Une inéquation fondamentale de la théorie de l'élasticité, Bull. Soc. Roy. Sci. Liege 3-4 (1962), 182-191.

[12] J. Gobert, Sur une inégalité de coercivité, J. Math. Anal. Appl. 36 (1971), $518-528$.

[13] H. Jia, D. Li, L. Wang, Regularity theory in Orlicz spaces for elliptic equations in Reifenberg domains, J. Math. Anal. Appl. 334 (2007), 804-817.

[14] R. V. Kohn, New estimates for deformations in terms of their strains, Ph. D. thesis, Princton (1979).

[15] S. Koizumi, On the singular integrals I, Proc. Japan Acad. 34 (1958), 193198.

[16] S. Koizumi, On the singular integrals II, Proc. Japan Acad. 34(1958), 235240.

[17] A. L. Krylov, Proof of Dirichlet's principle for the first boundary value problem of the nonlinear theory of elasticity, Dokl. Akad. Nauk. SSSR 146 (1962), 54-57.

[18] J. Málek, J. Nečas, M. Rokyta, M. Růžička, Weak and measure valued solutions to evolution partial differential equations, Appl. Math. and Math. Compl., 13, Chapman and Hall, London (1996).

[19] S. G. Mihlin, The problem of the minimum of a quadratic functional, GITTL, Moscow (1952).

[20] P. P. Mosolov, V. P. Mjasnikov, On the correctness of boundary value problems in the mechanics of continuous media, Math. USSR Sbornik 17 (2) (1972), 257-267.

[21] J. Nečas, Sur les normes équivalentes dans $W_{p}^{k}(\Omega)$ et sur la coecivité des formes formellement positives. In: Séminaire Equations aux Dérivées Partielles, Les Presses de l'Université de Montréal, 1966, 102-128.

[22] M. M. Rao, Z. D. Ren, Theory of Orlicz spaces, Marcel Dekker, New YorkBasel-Hongkong (1991).

[23] I. B. Simonenko, Boundedness of singular integrals in Orlicz spaces, Dokl. Akad. Nauk SSSR 130 (1960), 984-987.

[24] R. Temam, Mathematical problems in plasticity, Gauthier Villars, Paris (1985).

[25] A. Torchinsky, Interpolation of operations in Orlicz classes, Studia Math. 59 (1976/77), 177-207.

[26] E. Zeidler, Nonlinear Functional Analysis II/B-Nonlinear Monotone Operators, Springer Verlag, Berlin-Heidelberg-New York (1987).

\section{Martin Fuchs,}

Department of Mathematics,

Saarland University,

P.O. Box 1511 50,

D-66041 Saarbrücken, Germany

fuchs@math.uni-sb.de

Received on 26 March 2010. 